randomized were included in 5/6 reviews. ${ }^{2-7}$ The pair efficacy/ effectiveness was - with exceptions ${ }^{2} 5$ - included in four

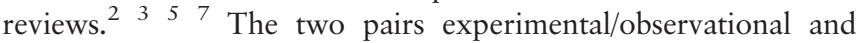
analytical/descriptive were included - with exceptions ${ }^{6}$ - each in two reviews ${ }^{4} 6$ The review by Schwartz \& Lellouch $^{2}$ included the pair of explanatory/pragmatic and discussed efficacy but not effectiveness. The CONSORT statement ${ }^{5}$ included three pairs of terms but did not discuss the meanings of efficacy/effectiveness. The remaining reviews ${ }^{3} 47$ included three but different pairs of terms. Our analysis of the studies confirmed the use of an experimental (RCT) design in 91\%, the assessment of the primary endpoint in $29 \%$, the assessment of efficacy in $14 \%$, of effectiveness in $25 \%$, and neither of these in $60 \%$. In $6 \%$ the study was classified as a pragmatic trial.

Conclusions The most likely reason for the observed incongruence is a lack of a consensus on terms and methods for reporting the results of pragmatic clinical trials. All reviews expect pragmatic trials to describe effects under RWC but assess these effects under experimental but not real-world conditions (RCTs) resulting in a conflict between expected and observed outcomes. CONSORT includes two imprecise statements. ${ }^{5}$ The review by Schwartz \& Lellouch $^{2}$ does not use the term 'randomized' and cannot justify the new name of 'randomized pragmatic trial'.5 Second, the aim of 2008 CONSORT is extension of its applicability to pragmatic trials. In fact, CONSORT changed 'pragmatic' into 'pragmatic randomised' and used an inappropriate reference ${ }^{2}$ to justify this change. The benefit of this maneuver is the recommendation of a valid instrument for assessment of effectiveness, but it may result in harm from the resulting confusion and incongruence of guideline recommendations.

\section{IS THE REVISED COCHRANE RISK OF BIAS TOOL RESEARCH READY FOR THE ERA OF OPEN SCIENCE AND PREREGISTRATION?}

${ }^{1}$ Merlin Bittlinger, ${ }^{2}$ Johannes Schwietering. ${ }^{1}$ Berlin Institute of Health, QUEST - Quality | Ethics | Open Science | Translation, Berlin, Germany; ${ }^{2}$ Hannover Medical School (MHH), Hannover, Germany

\subsection{6/bmjebm-2019-EBMLive.34}

Objectives Risk of bias (RoB) is an important to assess scientific evidence. Lack of detailed preregistration obfuscates reporting transparency and cast doubts about the required bijective relation between study protocol and the final scientific report of outcomes.

This contribution examines whether the lack of preregistration affects the judgments required for risk of bias assessment according to the RoB 2.0 tool and whether the tool can adequatly capture flaws in preregistration.

Method We examined the literature on RoB 2.0 and thoroughly evaluated the definition and criteria for the three different categories 'high risk of bias', 'low risk of bias', and some concerns of risk of bias'. Moreover, we investigated the literature on meta-science and performed a conceptual analysis of the epistemic merits and methodological benefits arising from various forms of preregistration. Accordingly, selective endpoint reporting, or endpoint modification raise serious and severe doubts about the scientific validity of biomedical randomized controlled trials (RCT) and preregistration is obligatory according to Article 35 of the Declaration of Helsinki of 2013.

Results The upcoming revised Cochrane handbook introduces RoB 2.0 as a new research tool for assessing the risk of bias of an RCT. RoB 2.0 requires a comparison between the prespecified analysis intentions and the reported analyses in order to assess potential selection bias of multiple outcomes or endpoints. In case a preregistered analysis plan is met, 'low risk bias' is assigned. 'High risk of bias' is assigned only if it is likely that reported outcomes have been selected based on the results, i.e. a deviation from the preregistered protocol is detected. If no information is available, RoB 2.0 suggests 'some concern'. Furthermore, in cases where preregistration is lacking, RoB 2.0 suggests the methods section of an article as a source of the analysis intentions. Therefore, the lack of preregistration does not by default lead to the evaluation of a 'high risk of bias'.

Conclusions Although lack of preregistration can lead to 'some concerns of risk of bias', there is by default no assignment of 'high risk of bias'even if a preregistration protocol is completely lacking. In light of the epistemic arguments in favour of preregistration, RoB 2.0 presents an untoward loophole in the risk of bias assessment with regard to selective outcome reporting or post hoc endpoint modification. Because any RoB 2.0 assessment is very effort-intensive and time-consuming, it is of utmost importance that all sort of biases are adequate considered and, thus, that future systematic reviews and metaanalysis benefit from risk of bias assessment tools that account for lack of preregistration as a source of 'high risk of bias' by default.

\section{SEX AND GENDER REPORTING AND ANALYSIS IN COCHRANE REVIEWS: A CROSS-SECTIONAL METHODS STUDY. PRELIMINARY RESULTS}

${ }^{1}$ Alba Antequera, ${ }^{1}$ Montserrat León, ${ }^{2}$ Sara Calderón, ${ }^{3}$ Ana Cuadrado. 'Iberoamerican Cochrane Centre - Sant Pau Biomedical Research Institute (IIB Sant Pau), Barcelona, Spain; ${ }^{2}$ Clinical Research Fellow at Queen Mary University of London, London, UK; ${ }^{3}$ London School of Hygiene and Tropical Medicine, London, UK

\subsection{6/bmjebm-2019-EBMLive.35}

Objectives Sex and gender health differences have been well established in the scientific literature. However, numerous studies present poor levels of inclusion of female participants and an insufficient sex/gender reporting and analysis. This lack of consideration of sex and gender in medical research reduces the applicability of findings and jeopardises its capacity to support informed decisions. Studies also suggest that women under-representation in science might be related to this deficient consideration of sex/gender in health research. This study aims to describe the degree of sex/gender reporting and analysis in Cochrane systematic reviews published in 2018 and investigate its association with gender of authorships.

Method Cross sectional study. We screened Cochrane reviews published in 2018. We removed reviews addressing sex-specific medical conditions and those that had been withdrawn by Cochrane. We collected data on gender and country affiliation of first and last authors, and review type. We documented the frequency of sex/gender terms used in each section of the reviews (abstract, methods, results, and discussion). In the 
results section, we split up descriptive information from primary studies (sex rates) and analytic approaches (considering sex/gender in the assessment of risk of bias, presenting disaggregated data by sex/gender or subgroup or heterogeneity analyses). We used 'not applicable' to denote a situation where insufficient primary studies or data on estimates did not enable to conduct the intended analyses (e.g. meta-analysis, subgroup analysis). We performed descriptive statistics and regression analyses to assess associations between authors' gender and sex/gender reporting.

Results 556 reviews were screened, of which 91 were excluded due to withdrawal $(19,8 \%)$ or sex-specific disease (80,2\%). Our analysis comprised 465 studies, including 2 prognosis, 4 methodology, 5 overview, 20 diagnostic and 421 intervention reviews. Women represented 53,8\% ( $\mathrm{n}=250)$ and $38,9 \%(\mathrm{n}=181)$ of first and last authorship, respectively, while in $25,3 \%$ of reviews both authors were women. $85,6 \%$ of authors belonged to high-income countries. 7.5\% ( $n=35)$ of reviews reported on sex in the abstract, 17.6\% ( $n=82)$ in the methods and 61.4\% $(\mathrm{n}=285)$ in the results section. Of these 285, 64.7\% provided descriptive results and 16,3\% had an analytic approach. In the discussion section, $13.5 \%$ (n $=63$ ) of reviews addresses sex-related findings. Only 4 studies scored positive in all 4 sections. Studies with female firstlast authorship had a non-significant increased probability of reporting on sex (RR [95\% CI] 1,24 [0.68-1.92])

Conclusions Consideration of sex and gender in Cochrane reviews is scarce. This prevents from generating inclusive and unbiased health research and inhibits effective population-wide translation of results. While women are less likely to be last authors, studies with a female first or last author show an increased (although non-significant) probability of reporting on sex.

\section{PSYCHOSOCIAL CONSEQUENCES OF INVITATION TO COLORECTAL CANCER SCREENING - A DANISH LONGITUDINAL COHORT STUDY}

\footnotetext{
1,2 2 essica Malmqvist, 'Volkert Siersma, 'Mie Sara Hestbech, 'Dagný Rós Nicolaisdóttir, ${ }^{3}$ Christine Winther Bang, ${ }^{2,3}$ John Brodersen. ${ }^{1}$ Center for Research and Education in General Practice, Copenhagen, Denmark; 'Research Unit for General Practice in Region Zealand, Copenhagen, Denmark; ${ }^{3}$ The Center for Research and Education in General Practice, Copenhagen, Denmark
}

\subsection{6/bmjebm-2019-EBMLive.36}

Objectives Harms of screening are generally not well reported. Harms of colorectal cancer (CRC) screening may include negative psychosocial consequences from the attention being drawn to disease through the screening invitation, the screening procedure itself and from fear of the screening result. Hence, it is important to measure psychosocial consequences before and after invitation to screening, at screening and after screening. Moreover, measurement of psychosocial consequences in cancer screening settings should be performed using questionnaires with high content validity and adequate measurement properties. Despite this fact, few CRC screening studies investigating psychosocial consequences have performed a baseline measurement before and after invitation to screening in both a screening and a control cohort. Moreover, none of the studies has used a questionnaire with high content validity and adequate measurement properties.

Therefore, the objective of this study was to investigate the psychosocial consequences of invitation to CRC screening in a
Danish CRC screening naïve cohort using a condition-specific questionnaire validated with Item Response Theory Rasch Models.

Method A random sample of 1000 screening invitees scheduled for screening in March 2017 was matched with 1000 control persons scheduled for screening in November and December 2017. We sent a questionnaire to both study groups five weeks prior to the invitation of the invitees and another questionnaire to both groups three days after the invitees' screening invitations were sent by post.

The primary outcome was psychosocial status, measured with the condition-specific questionnaire Consequences of Screening for CRC (COS-CRC).

We analysed the mean COS-CRC score at each measurement for each COS-CRC scale compared between the study groups using multivariable regression models, adjusting for potential confounders. We adjusted for differential nonresponse by weighting the observations that were available at the follow-up measurement by the inverse of the probability of not being missing. We also adjusted for repeated measures and weighting. To allow for multiple testing, a p-value $<0.01$ was considered significant.

Results Results and conclusions will be presented at the conference.

Conclusions Results and conclusions will be presented at the conference.

\section{INCIDENCE OF SUBSTUDIES PUBLICATION AFTER ORIGINALLY NEGATIVE CLINICAL TRIALS AND RATE OF POSITIVATION: A CALL FOR SCIENTIFIC INTEGRITY}

1,2Luis Correia, 'Diego Rabelo, 'Alessandra Caldas, 'Bruno Góes, 'Janine Magalhães, ${ }^{3}$ Daniel Medina, 'Denise Matias. 'Bahiana Medical and Public Health School, Salvador, Brazil; ${ }^{2}$ Hospital São Rafael, D'Or Institute for Research and Education, Salvador, Brazil; ${ }^{3}$ School of Medicine, Federal University of Bahia, Santo Antonio de Jesus, Brazil

\subsection{6/bmjebm-2019-EBMLive.37}

Objectives After a negative study, substudies may emerge providing a positive spin on the hypothesis, based on subgroup or secondary outcome analyses. This approach suffers from multiplicity problem, imprecision and higher risk of type I error.

To describe the incidence of substudies testing the same hypothesis over a period of 5 years after publication of a primarily negative randomized clinical trial; to access the most frequent subanalysis method, how explicit the exploratory nature of the analysis were and the rate of successful positivation of results.

Method We searched all negative randomized trials published in the New England Journal of Medicine (NEJM) during the year 2014. Then, we made a highly sensible PUBMED search over the following 5 years in order to detect subsequent articles testing the same hypothesis in the same dataset throughout secondary analysis. Methods utilized, lack of recognition as secondary analysis was described and rate of positivation were described.

Results During the year 2014, 46 negative randomized clinical trials were published in the NEJM. Over five years, 14 of those articles had subsequent publication of subanalysis in PUBMED indexed journals, an incidence of 30\% $(95 \% \mathrm{CI}=$ $19 \%$ - 45\%). The most frequent method was secondary endpoints analysis (67\% of substudies) and the remaining made use of subgroup analysis. Half of those analysis were not 